\title{
Doctors' responses to patients' concerns; an exploration of communication sequences in gynaecology
}

\author{
SANDRA VAN DULMEN', MATTHIAS NÜBLING², WOLF LANGEWITZ³ \\ ' NIVEL (Netherlands Institute of Health Services Research), Utrecht, The Netherlands \\ ? Society for Empirical Consulting (GEB), Freiburg i Brsg., Germany \\ ${ }^{3}$ Division Psychosomatic Medicine, Department of Internal Medicine, University Hospital Basel, Basel, Switzerland
}

\begin{abstract}
SUMMARY. Aims - Like other medical doctors, gynaecologists have difficulty attending to psychosocial issues and concerns. Communication training has proven to be effective in teaching them to spend more time on discussing these factors. However, whether or not they do this in response to patients' utterances remains unclear. The question is how gynaecologists respond to patients' concerns, whether or not they do this adequately and what the effects of a communication training are on the use of these communication sequences. Methods - Nineteen gynaecologists participated in a study which examined the effects of a three-day residential communication training. Before and after the training the gynaecologists videotaped series of consecutive outpatient visits. The communication during these visits was rated using the Roter Interaction Analysis System. Gynaecologists' responses to patients' concerns were examined at lag one, i.e. immediately following the patient's concern. Results - The most prevalent responses made by the gynaecologists were showing agreement and understanding and giving medical information. Affective responses were observed less. At postmeasurement, the gynaecologists responded neither more adequately nor inadequately to patients' concerns. Conclusions - The gynaecologists did not respond in a very affective way to patients' concerns. However, the patients did not express many concerns. Future studies should focus on more prevalent communication behaviours and incorporate more lags.
\end{abstract}

Declaration of Interest: none.

KEY WORDS: concems, gynaecology, communication sequences, communication training.

\section{INTRODUCTION}

Because of its potential non-specific, diagnostic and therapeutic effects, communication is generally considered as a powerful tool in health care. Yet, by virtue of this power, these effects may just as much be detrimental as beneficial. After all, failing to communicate effectively can have a large impact on visit and patient outcome in terms of non-adherence, dissatisfaction and second opinion seeking (Mellink et al., in press).

Several studies suggest that communication problems more often occur in relation to physicians' skills in adequately responding to patients' expressions of emotions (anger, fear or worry) than to their educational skills. Apart from exploring the expressed emotion, physicians often start to reassure a patient immediately or negate the emotion all together (Weijts et al., 1991). As a result, emotional distress often goes unrecognized (Del Piccolo et al., 2000) and many patients leave the consulting room

Address for correspondence: Dr. A.M. van Dulmen, NIVEL, P.O.Box 1568, 3500 BN Utrecht (The Netherlands).

Fax: $++31-302729729$

E-mail: S.vandulmen@nivel.nl not feeling relieved and understood. Patients' concerns therefore deserve more attention.

In gynaecology, attention to patients' concerns is especially warranted. After all, gynaecological disorders often coincide with emotional or marital problems. Some patients may, for instance, worry about the level to which the gynaecological disorder interferes with their marital life while others feel distressed about their presumed reproductive incapacity (Hunter, 1990). Patients like their gynaecologist to take notice of these feelings (Himmel $e t$ al., 1999). Yet, the gynaecological curriculum is primarily directed at acquiring technical medical skills. Postgraduate education may be necessary to teach gynaecologists to handle these issues more adequately.

In 1997, a three-day training course was held to improve the way gynaecologists communicate with their patients about their worries and concerns. Comparison of the communication process before and after the training showed that, at post-measurement, gynaecologists talked more often about psychosocial issues without lengthening the outpatient visit (van Dulmen \& van Weert, 2001). Although at first glance this may seem to be rather positive results, it still does not make clear whether or not gynaecologists really respond more adequately to patients' concerns or only initiate a discussion about psychosocial issues irrespective of the content of the 
patient's talk. Like in most communication studies, only frequencies of communication behaviours were counted which ignores the role of the patients. To incorporate patients' contribution, sequences of communication behaviours, i.e. what physician responses follow patient utterances, will have to be investigated.

An important aim of the training course was to increase the gynaecologists' skills to respond to patients' concerns adequately. By responding adequately, the gynaecologist acknowledges the affective load of the patient's expression and either facilitates further disclosure by giving backchannel responses or paraphrases or responds in an affective way by providing reassurances or empathic responses. Inadequate responses ignore the affective load by continuing talking about medical issues. This paper focuses on how gynaecologists respond to the concerns expressed by the patients by examining the following questions:

1. With what kind of responses do gynaecologists react to patients' concerns?

2. How many of these responses can be defined as adequate and inadequate?

3. Do gynaecologists differ in the number of adequate and inadequate responses?

4. Does a communication training course influence the number of adequate and inadequate utterances expressed by gynaecologists in response to patients' concerns?

\section{METHODS}

\section{Sample}

A total of 21 gynaecologists participated in the study which consisted of videotaping two series of consecutive outpatient visits and participating in the three-day residential training course (van Dulmen, 1999). The gynaecologists completed the training in two groups. At postmeasurement, two residents had changed workplaces. So, the effects of the training were measured in 19 gynaecologists. Prior to and four months after completing the training, all participants were asked to videotape a series of general gynaecology outpatient visits. About one week before the appointment with the gynaecologists, patients were informed of the nature of the study and were asked to give written consent to allow recording for research purposes. Finally, the 19 gynaecologists videotaped 557 outpatient visits using a fixed unmanned camera, 291 at pre-measurement and 266 at post-measurement. Patients attended the gynaecologists with either gynaecological problems or obstetric complications requiring specialist supervision. Training effects were measured by comparing gynaecologists' performance on the videotapes at pre- and post-measurement. The use of videotapes to evaluate physician performance in actual medical visits has proved to be a reliable measure (McCormick et al., 1993). The study was approved by the Medical-Ethical Committee of the University Hospital Maastricht as well as by the local committees of the participating hospitals. In the Netherlands a gynaecologist is a consulting physician and member of a hospital's staff.

\section{Communication training}

Two experienced trainers, a psychotherapist and a primary care physician, ran all the meetings of the communication training. The training aimed at identifying maladaptive communication skills (e.g. ignoring nonverbal signals) and encouraging and acquiring alternative and more effective ways of communicating with patients (e.g. inquiring about patients' feelings). These aims were accomplished by assisting gynaecologists to acquire patient-centred interviewing skills (Levenstein et al, 1986) (i.e., skills needed to unravel patients' experience of illness) and to handle patients' instrumental need (i.e., the need to know and understand what is wrong) and affective need (the need to feel known and understood by the doctor) by providing information and advice and showing support and understanding, respectively. An important part of this experiential training was dedicated to watching and discussing videotapes of trainees' actual performance during outpatient encounters. These discussions focused on real-life examples of effective and less effective communication behaviours. This gave insight into each gynaecologist's characteristic way of communicating and encouraged a learner-centred approach with personal learning objectives, self-awareness, colleagues' constructive feedback and comments, and suggestions for alternatives.

In sum, the training combined:

1. Gynaecologist education on the significance of a therapeutic doctor-patient relationship, of communicating affect (verbally and nonverbally), of attending to psychosocial issues, and of giving the patient room to talk

2. Theoretical and practical homework and application of what was learned between sessions

3. Role-playing exercises, which provided an opportunity of becoming comfortable with alternative communication skills

4. Trainers' and colleagues' feedback of videotaped and role-play interaction style

5. Discussion of gynaecologists' own experiences

These training techniques have been identified as edu- 
cational methods of demonstrated effectiveness and feasibility (Roter et al., 1990; 1995). In terms of the aims of the training, trainees were expected to become less verbally dominating and more supportive; and to pay more attention to patients' thoughts and feelings, as dictated by a patient-centred approach and to look at the patient more often. The training was accredited by the Dutch Society of Obstetrics and Gynaecology.

\section{Communication categories}

The verbal and nonverbal communication between gynaecologist and patient was measured by four independent raters directly from the video-recordings.

Verbal behaviour. The verbal communication process was analysed using an adjusted version of the Roter Interaction Analysis System (RIAS), which is specially designed to code both doctor and patient communication (Roter, 1989). This system distinguishes instrumental or task-related and affective or socio-emotional verbal utterances by doctors and patients. Each statement that is made during the medical encounter by either the physician or the patient can be assigned to one of these mutually exclusive and exhaustive categories. Instrumental categories refer to those communication aspects which primarily focus on solving problems, such as giving information, asking questions, and counselling in medical or psychosocial topics. Affective categories refer to those aspects needed to establish a therapeutically effective relationship between the interactants, such as giving comfort and reassurance and showing optimism, concern and understanding. The content and discriminant validity of the RIAS prove to be satisfactory (Ong et al., 1998). Based on the findings from earlier studies, the categories were reduced to sixteen clusters identical for gynaecologist and patient (Bensing, 1991; van Dulmen et al., 1997). Patients' concerns are defined as statements or non-verbal expressions indicating that a condition or event is serious, worrisome, distressing or deserving special attention and is of particular concern right now during the medical visit. In other words, these statements have a strong and immediate emotional or psychosocial component and do not refer to a more general frame of mind or past issues (Roter, 1989).

\section{Communication sequences}

In this paper we aim to examine how gynaecologists respond to patients' concerns, and whether or not these responses are adequate. So, the focus of attention is on communication sequences. As sequence we consider each two-utterance chain consisting of a patient concern as initial utterance followed by the very next utterance of the gynaecologist, i.e., at lag one. In conformity with others (van den Brink-Muinen \& Caris-Verhallen, 2003), adequate responses were defined as agreements, paraphrases, reflections, concerns or reassurances expressed by the gynaecologists and orientations, questions, information giving and counselling as their inadequate responses (table I).

\section{RESULTS}

First, it was examined how the gynaecologists in this study responded to the patients' concerns. Taken together, the gynaecologists expressed a total of 160719 utterances. These 160719 utterances led to $160718 \mathrm{com}$ munication sequences in which one utterance is followed by an utterance of the other interactant. A total of 1210 sequences $(0.75 \%)$ could be identified in which a patient concern is expressed and followed by a gynaecologist response. Figure 1 shows the frequencies of the different responses made by the gynaecologists in descending order. The most prevalent responses were agreements, followed by medical responses, such as providing medi-

Table I. - Gynaecologists' adequate and inadequate responses to patients' concerns.

\begin{tabular}{|c|c|}
\hline \multicolumn{2}{|c|}{ Adequate responses } \\
\hline Agreements & Signs of agreement or understanding, backchannel responses \\
\hline Paraphrases & Restatements of information told by the other \\
\hline Reflections & Empathic statements which name the other's emotional state \\
\hline Concerns & Statements which indicate that a condition is serious or worrisome \\
\hline Reassurances & Statements which indicate optimism, encouragement, relief or reassurance \\
\hline \multicolumn{2}{|c|}{ Inadequate responses } \\
\hline Orientations & Directive remarks regarding the medical visit process \\
\hline Questions & $\begin{array}{l}\text { Questions that ask for information on medical condition, therapeutic regimen, lifestyle, family or home situations, } \\
\text { stress or personal likes or dislikes }\end{array}$ \\
\hline Information & Statements or facts relating to medical condition, diagnosis, test results, lifestyle, family or home situations or stress \\
\hline Counselling & $\begin{array}{l}\text { Statements which suggest resolution or action to be taken by the other relating to medical condition, diagnosis, test } \\
\text { results, lifestyle, family and home situations or stress }\end{array}$ \\
\hline
\end{tabular}


cal information and asking medical questions. Affective responses can be noticed at the sixth position.

Of all these gynaecologists' responses, more than half could be identified as adequate and one third as inadequate responses (table II).

The gynaecologists who participated in this study showed large variability in responding more or less adequately to patients' concerns (figure 2). In six gynaecologists the number of inadequate responses exceeded the number of adequate responses.

Table III shows the frequencies of the gynaecologists' adequate and inadequate responses before and after atten-

Table II. - Frequency (\%) of gynaecologists' responses to patients' concerns.

\begin{tabular}{|c|c|c|c|}
\hline & & $\mathrm{n}$ & (\%) \\
\hline \multicolumn{4}{|c|}{ Adequate responses } \\
\hline & Agreements & 571 & (47.2) \\
\hline & Paraphrases & 74 & $(6.1)$ \\
\hline & Reflections & 43 & (3.5) \\
\hline & Concerns & 12 & $(0.9)$ \\
\hline & Reassurances & 38 & (3.1) \\
\hline subtotal & & 738 & $(60.9)$ \\
\hline \multicolumn{4}{|c|}{ Inadequate responses } \\
\hline & Orientations & 67 & $(5.5)$ \\
\hline & Questions & 116 & $(9.6)$ \\
\hline & Information & 209 & (17.3) \\
\hline & Counselling & 26 & $(2.1)$ \\
\hline subtotal & & 418 & (34.5) \\
\hline \multicolumn{4}{|c|}{ Other responses } \\
\hline subtotal & & 54 & (4.5) \\
\hline total & & 1210 & $(100)$ \\
\hline
\end{tabular}

ding the training course. No significant differences were found between the two measurements. In other words, after the training the gynaecologists responded neither

Figure 1. - Gynaecologists' responses to patients' concerns ( $n=1210)$.

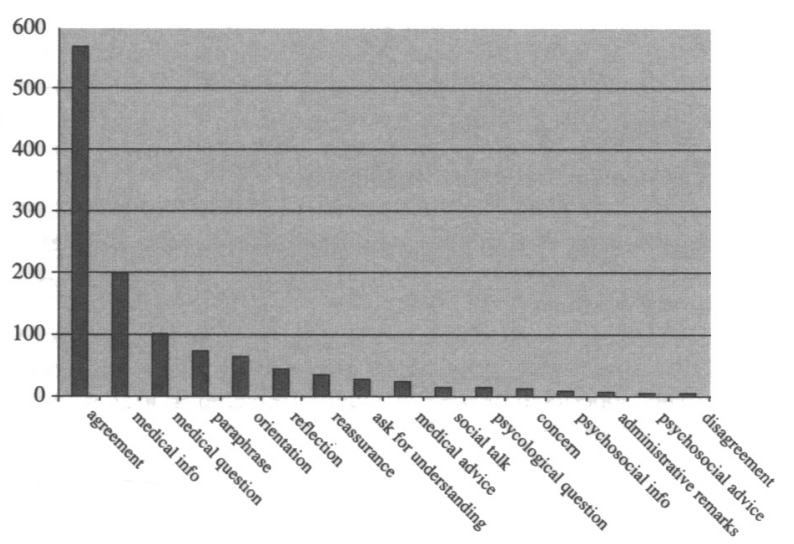

more adequately nor more inadequately to the patients' concerns.

Figure 2. - Mean percentages of adequate and inadequate responses for the different gynaecologists.

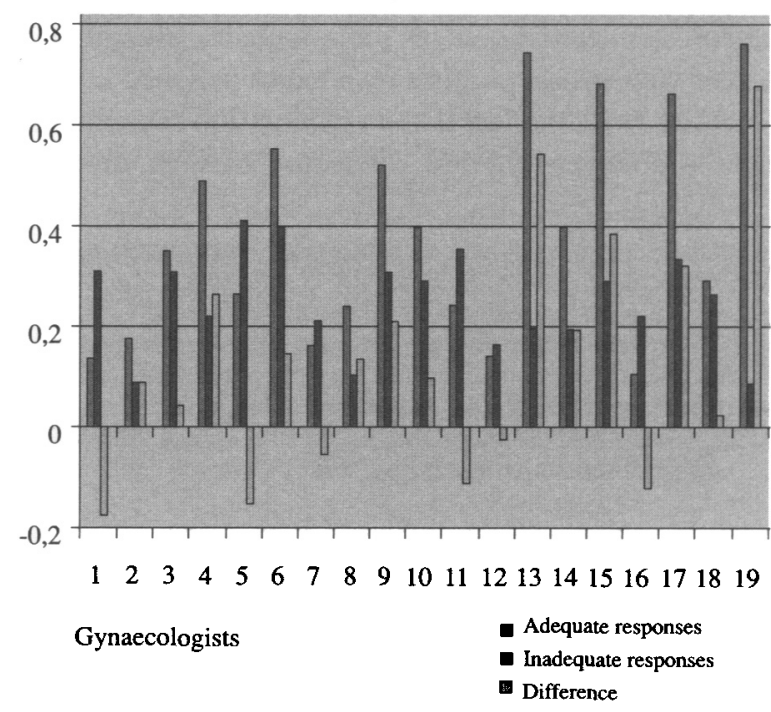

\section{DISCUSSION}

The present study shows that gynaecologists use a wide range of communication behaviours in response to patients' concerns. The most prevalent ones, expressions of agreement and understanding, are primarily aimed at facilitating patients' disclosure. Although this kind of responses might be quite beneficial in this respect, they do ignore the emotional load of the patients' talk. Such really affective responses occur quite seldom. When the gynaecologists pay attention to the content of the concerns expressed by the patients, they tend to do this most often by elaborating on medical issues, thereby again ignoring the emotional content.

Contrary to our former study in which we presented the, quite positive, effects of a communication training for gynaecologists (van Dulmen \& van Weert, 2001), the present results using the same data look somewhat disappointing. After all, no changes were found in gynaecologists' responses to patients' concerns, which was an important aim of the training course. Yet, patients' concerns, i.e. the focus of our attention, were quite rare. For studying sequences the way we did, it may be necessary to focus attention on more prevalent communication behaviours such as the exchange of medical information. Post hoc analysis indeed shows that the gynaecologists expressed no adequate responses in 323 visits (58\%) but

Epidemiologia e Psichiatria Sociale, 12, 2, 2003 
also no inadequate responses in 334 visits $(60 \%)$, which is to a large extent caused by the fact that patients quite sporadically express their concerns in a verbal way. Nevertheless, given the rather instrumental content of gynaecologists' responses made to the patients' concerns, in communication courses more attention should be paid to the way gynaecologists respond to patients' concerns.

Apart from the fact that an exploration of communication sequences provide different findings than when studying frequencies, it must be remembered that in this study only lag one sequences were studies. Other gynaecologists' utterances at lag two or three were not accounted for. Still, these utterances could also be made in response to the patients' concerns. In future studies it might be worthwhile to incorporate other lags as well, as this will provide a more complete picture of gynaecologists responses.

Table III. - Frequency (\%) of gynaecologists' adequate and inadequate responses to patients' concerns at pre-and post-intervention.

\begin{tabular}{llllll}
\hline & \multicolumn{2}{c}{ Pre-intervention } & \multicolumn{2}{c}{ Post-intervention } \\
& & $\mathrm{n}$ & $(\%)$ & $\mathrm{n}$ & $(\%)$ \\
\hline Adequate responses & & & & \\
& Agreements & 310 & $(51.0)$ & 261 & $(47.1)$ \\
& Paraphrases & 30 & $(5.0)$ & 44 & $(7.9)$ \\
& Reflections & 25 & $(4.2)$ & 18 & $(3.2)$ \\
& Concerns & 5 & $(0.8)$ & 7 & $(1.3)$ \\
& Reassurances & 20 & $(3.3)$ & 18 & $(3.2)$ \\
subtotal & 390 & $(64.8)$ & 348 & $(62.8)$ \\
& & & & & \\
Inadequate & responses & 36 & $(5.9)$ & 31 & $(5.6)$ \\
& Orientations & 64 & $(10.6)$ & 52 & $(9.4)$ \\
& Questions & 98 & $(16.3)$ & 111 & $(20.0)$ \\
& Information & 14 & $(2.3)$ & 12 & $(2.2)$ \\
Subtotal & Counselling & 212 & $(35.2)$ & 206 & $(37.2)$ \\
\hline total & & 602 & $(100)$ & 554 & $(100)$ \\
\hline
\end{tabular}

\section{REFERENCES}

Bensing J.M. (1991). Doctor-patient Communication and the Quality of Care. (PhD thesis). NIVEL: Utrecht.

Brink-Muinen van den A. \& Caris-Verhallen W. (2003). Doctors' responses to patients' concerns; testing the use of sequential analysis. Epidemiologia e Psichiatria Sociale, 12, 92-97.

Del Piccolo L., Saltini A., Zimmermann C. \& Dunn G. (2000). Differences in verbal behaviours of patients with and without emotional distress during primary care consultations. Psychological Medicine 30, 629-643.

Dulmen van A.M. (1999). Communication during gynecological outpatient consultations. Journal of Psychosomatic Obstetrics and Gynecology 20, 119-126.

Dulmen van A.M. \& Weert van J.C.M. (2001). Effects of gynaecological education on interpersonal communication skills. British Journal of Obstetrics and Gynaecology 108, 485-491.

Dulmen van A.M., Verhaak P.F.M. \& Bilo H.J.G. (1997). Shifts in doctor-patient communication during a series of outpatient consultations in non-insulin-dependent diabetes mellitus. Patient Education and Counseling 30, 227-237.

Himmel W., Ittner E., Kron M. \& Kochen M.M. (1999). Comparing women's views on family and sexual problems in family and gynecological practices. Journal of Psychosomatic Obstetrics and Gynecology 20, 127-135.

Hunter M. (1990). Gynaecological complaints. In Somatization: Physical Symptoms \& Psychological lllness (ed. C.Bass). Blackwell Scientific Publishers: Oxford.

Levenstein J.H., McCracken E.C., McWhinney I.R., Stewart M.A. \&
Brown J.B. (1986). The patient-centred clinical method. 1. A model for the doctor-patient interaction in family medicine. Family Practice 3, 24-30.

McCormick D.P., Rassin G.M., Stroup-Benham C.A., Baldwin C.D., Levine H.G., Persaud D.I. \& Wolf W.J. (1993). Use of videotaping to evaluate pediatric residents performance of health supervision examinations of infants. Pediatrics 92, 116-120.

Mellink W.A.M., Dulmen van A.M., Wiggers Th., Spreeuwenberg P.M.M., Eggermont A.M.M. \& Bensing J.M. (in press). Cancer patients seeking a second opinion: results of a study on motives, needs and expectations. Journal of Clinical Oncology.

Ong L.M.L., Visser M.R.M., Kruyver I.P.M., Bensing J.M., van den Brink-Muinen A., Stouthard J.M., Lammes F.B. \& de Haes J.C. (1998). The Roter Interaction Analysis System (RIAS) in oncological consultations: psychometric properties. Psycho-Oncology 7 , 387-401.

Roter D. (1989). The Roter Method of Interaction Process Analysis. Johns Hopkins University: Baltimore:

Roter D.L., Cole K.A., Kern D.E., Barker L.R. \& Grayson M. (1990). An evaluation of residency training in interviewing skills and the psychosocial domain of medical practice. Joumal of General Internal Medicine 5, 347-354.

Roter D.L., Hall J.A., Kern D.E., Barker L.R., Cole K.A. \& Roca R.P. (1995). Improving physicians' interviewing skills and reducing patients' emotional distress. A randomized clinical trial. Archives of Internal Medicine 155, 1877-1884.

Weijts W., Widdershoven G. \& Kok G. (1991). Anxiety-scenarios in communication during gynaecological consultations. Patient Education and Counseling 18, 149-163. 